\title{
Prediction of nuclear proteins using nuclear translocation signals proposed by probabilistic latent semantic indexing
}

Emily Chia-Yu Su${ }^{1 *}$, Jia-Ming Chang ${ }^{2,3}$, Cheng-Wei Cheng ${ }^{4}$, Ting-Yi Sung ${ }^{4}$, Wen-Lian Hsu ${ }^{4}$

From Asia Pacific Bioinformatics Network (APBioNet) Eleventh International Conference on Bioinformatics (InCoB2012)

Bangkok, Thailand. 3-5 October 2012

\begin{abstract}
Background: Identification of subcellular localization in proteins is crucial to elucidate cellular processes and molecular functions in a cell. However, given a tremendous amount of sequence data generated in the postgenomic era, determining protein localization based on biological experiments can be expensive and timeconsuming. Therefore, developing prediction systems to analyze uncharacterised proteins efficiently has played an important role in high-throughput protein analyses. In a eukaryotic cell, many essential biological processes take place in the nucleus. Nuclear proteins shuttle between nucleus and cytoplasm based on recognition of nuclear translocation signals, including nuclear localization signals (NLSs) and nuclear export signals (NESs). Currently, only a few approaches have been developed specifically to predict nuclear localization using sequence features, such as putative NLSs. However, it has been shown that prediction coverage based on the NLSs is very low. In addition, most existing approaches only attained prediction accuracy and Matthew's correlation coefficient (MCC) around 54\% 70\% and 0.250 0.380 on independent test set, respectively. Moreover, no predictor can generate sequence motifs to characterize features of potential NESs, in which biological properties are not well understood from existing experimental studies.

Results: In this study, first we propose PSLNuc (Protein Subcellular Localization prediction for Nucleus) for predicting nuclear localization in proteins. First, for feature representation, a protein is represented by gappeddipeptides and the feature values are weighted by homology information from a smoothed position-specific scoring matrix. After that, we incorporate probabilistic latent semantic indexing (PLSI) for feature reduction. Finally, the reduced features are used as input for a support vector machine (SVM) classifier. In addition to PSLNuc, we further identify gapped-dipeptide signatures for putative NLSs and NESs to develop a prediction method, PSLNTS (Protein Subcellular Localization prediction using Nuclear Translocation Signals). We apply PLSI to generate gappeddipeptide signatures from both nuclear and non-nuclear proteins, and propose candidate sequence motifs for putative NLSs and NESs. Then, we incorporate only the proposed gapped-dipeptide signatures in an SVM classifier to mimic biological properties of NLSs and NESs for predicting nuclear localization in PSLNTS.
\end{abstract}

Conclusions: Experiment results demonstrate that the proposed method shows a significant improvement for nuclear localization prediction. To compare our predictive performance with other approaches, we incorporate two non-redundant benchmark data sets, a training set and an independent test set. Evaluated by five-fold crossvalidation on the training set, PSLNuc attains an overall accuracy of $79.7 \%$, which is $4.8 \%$ improvement over the state-of-the-art system. In addition, our method also enhances the MCC from 0.497 to 0.595 . Compared on the

\footnotetext{
* Correspondence: emilysu@tmu.edu.tw

${ }^{1}$ Graduate Institute of Biomedical Informatics, Taipei Medical University,

Taipei, Taiwan

Full list of author information is available at the end of the article
} 


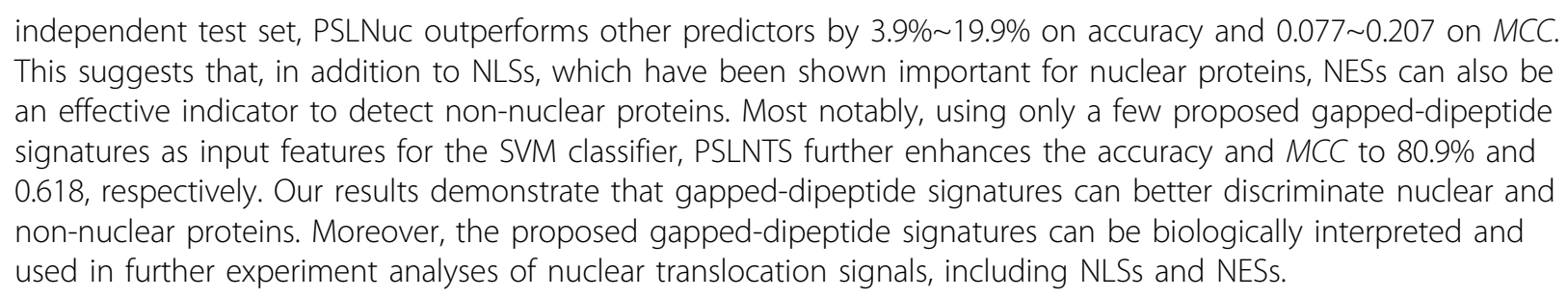

\section{Background Introduction}

In the eukaryotic cells, many essential biological processes take place in the nucleus. Nuclear localization is a complicated set of processes that play a crucial role in the dynamical self-regulation of the cell [1]. To participate in the cell regulation processes, proteins are translocated in and out of nucleus. This import and export are mediated by short binding sites on the protein sequence, called nuclear localization signals (NLSs) and nuclear export signals (NESs). Both NLSs and NESs have been used as important features to detect nuclear proteins. However, due to a tremendous amount of protein sequences generated from the post-genomic era, NLSs and NESs are not yet well understood from existing experiments by the biologists, and so the set of currently known NLSs and NESs may be incomplete. Therefore, developing computational methods to identify potential NLSs and NESs has become highly desirable to predict nuclear localization.

\section{Previous works}

At present, only a few predictors are designed specifically to identify proteins imported into the nucleus. PredictNLS [2] predicts nuclear proteins based on the presence of known or putative NLSs derived from the contents of NLSdb. NucPred [3] uses regular expression matching and multiple program classifiers induced by genetic programming to detect putative NLSs. NUCLEO [4] incorporates sequence motifs from known NLSs in a support vector machine (SVM) classifier for predicting nuclear localization. NpPred [5] applies SVM classifiers and hidden Markov models (HMM) using $k$-peptide composition and achieves high accuracy based on a data set, in which proteins are filtered at $90 \%$ sequence identity [6]. Although general localization prediction methods provide comprehensive information, they do not consider compartmentspecific features to optimize for a particular localization site [5]. Besides the above predictors designed to predict nuclear localization proteins, several methods, such as NLStradamus, NetNES, and NoD, have been proposed to detect NLSs and NESs. NLStradamus [7] uses HMMs to predict NLSs in proteins, and NetNES [8] predicts NESs using neural network and HMMs. In addition, NoD [9] applies artificial neural network algorithm to detect nucleolar localization sequences in eukaryotic and viral proteins. Moreover, several methods [10-13] have been developed to further classify nuclear proteins according to their subnuclear localizations. In this study, we will propose a method to improve nuclear localization prediction based on potential NLSs and NESs generated from our analysis of gapped-dipeptide signatures.

\section{Challenges}

Prediction of nuclear proteins presents several challenges. First, methods that integrate biological features only from known or putative NLSs could suffer from the problem of low coverage in high-throughput proteomic analyses due to the lack of information to characterize NESs from nuclear exported proteins. Second, several predictors are implemented on redundant training sets, which might lead to overestimation of the predictive performance. Thus, the performance would be significantly lower if redundant sequences were meticulously removed (e.g., at $25 \%$ sequence identity or even less) [4]. Meanwhile, the performance of amino acid composition-based and sequence homology-based methods might be significantly degraded if homologous sequences are not detected [14]. In addition, the $k$-peptide feature representation from amino acid composition-based methods can result in a very large feature dimension during the machine learning procedure, in which an effective feature reduction is highly desirable to reduce dimension. Finally, results of these two types of methods are generally difficult to interpret; therefore, it is difficult to determine which biological features should be used to identify nuclear or non-nuclear proteins and why they work well for prediction. If the features were biologically interpretable, the resultant knowledge could help in designing artificial proteins with the desired properties.

\section{Our contributions}

In this study, we first present a method, PSLNuc (Protein Subcellular Localization prediction for Nucleus), for predicting nuclear localization in proteins. For feature representation, sequence homology information from a smoothed position-specific scoring matrix (PSSM) is incorporated to calculate the weights of gapped-dipeptides. 
After that, probabilistic latent semantic indexing (PLSI) is used for feature reduction. Finally, the reduced features are applied as input vectors for an SVM classifier. In addition to PSLNuc, we further generate gapped-dipeptide signatures for potential NLSs and NESs, and develop another prediction method, PSLNTS (Protein Subcellular Localization prediction using Nuclear Translocation Signals). To propose candidate sequence patterns of putative NLSs and NESs, we apply PLSI to generate gapped-dipeptide signatures from both nuclear and non-nuclear proteins. Then, we further incorporate only the proposed gapped-dipeptide signatures in an SVM classifier to mimic biological properties of NLSs and NESs in PSLNTS.

Experiment results show that PSLNuc achieves high prediction accuracy, which demonstrates that homology information of gapped-dipeptides reduced by PLSI can significantly enhance the performance. Our analysis suggests that, in addition to NLSs, which have been shown important for nuclear proteins, NESs could also be an effective indicator to detect non-nuclear proteins. Most notably, the overall accuracy of PSLNTS is further improved to 0.809 using only the proposed gapped-dipeptide signatures. This implies that gapped-dipeptide signatures can better discriminate nuclear and non-nuclear localization. In addition, since sequence redundancy tends to overestimate the predictive performance, we incorporate nonredundant data sets and show the general accuracy of nuclear prediction should be approximately 0.800 . Finally, since the proposed gapped-dipeptide signatures are biologically interpretable, they can be easily applied to advanced analyses and experimental designs of nuclear translocation signals.

\section{Results and discussion}

\section{Data sets}

To compare the predictive performance with other approaches, we utilize two benchmark data sets of proteins from Swiss-Prot that have been constructed in previous works [2-4]. The training and testing sets are comprised of proteins whose localization sites are experimentally determined. In addition, both the nuclear and non-nuclear proteins are redundancy-reduced using BlastClust with a $10 \%$ identity threshold, so that the remaining sequences have no more than $10 \%$ identical residues in the aligned regions covering at least $90 \%$ of the sequences. Table 1 lists the number of nuclear and nonnuclear proteins in the training and testing data sets. The training and testing sets are available in the supplementary material [see Additional file 1].

\section{Predictive performance on benchmark data sets}

Table 2 shows the performance comparison with other approaches based on two benchmark data sets, a training set and an independent test set. First, evaluated by a five-
Table 1 Data sets

\begin{tabular}{ccc}
\hline & Nuclear & Non-nuclear \\
\hline Training & 2,842 & 2,606 \\
Testing & 564 & 398 \\
\hline Total & 3,406 & 3,004 \\
\hline
\end{tabular}

Numbers of nuclear and non-nuclear proteins for training and testing.

fold cross-validation on the training set, PSLNuc attains an overall accuracy of 0.797 , which is a $4.8 \%(0.048)$ improvement over the state-of-the-art performance by NUCLEO. In addition, our method also enhances the Matthew's correlation coefficient (MCC) from 0.497 to 0.595 . Secondly, an independent test data set is incorporated to compare the predictive performance of PSLNuc, NUCLEO, PredictNLS, and NucPred. For the overall accuracy, PSLNuc significantly outperforms the other approaches by $3.9 \%(0.039)$ to $19.9 \%(0.199)$. Moreover, our method performs better by 0.077 to 0.207 in terms of $M C C$. Experiment results demonstrate that feature reduction by PLSI is able to extract discriminative features for predicting nuclear localization. Meanwhile, our method suggests that proposed smoothed PSSM (smoothPSSM) weighting scheme can better discriminate nuclear and non-nuclear localization by the incorporation of neighboring residues. Finally, in addition to NLSs, which have been shown important for nuclear proteins, NESs could also be an effective indicator to detect non-nuclear proteins.

\section{Proposed gapped-dipeptide signatures correspond well with known nuclear translocation signals}

To generate gapped-dipeptides for nuclear and nonnuclear localization, we choose ten preferred topics for nuclear and non-nuclear proteins based on localizationpreference confidence, respectively. The confidence is calculated by the absolute value of nuclear localizationpreference minus non-nuclear localization-preference. For every preferred topic, we select up to twenty most abundant gapped-dipeptides. Finally, the resultant gapped-dipeptide signatures for nuclear and non-nuclear

Table 2 Performance comparison

\begin{tabular}{lcccccccc}
\hline \multirow{8}{*}{ Method } & \multicolumn{7}{c}{ Training } & Data Set (by five-fold cross-validation) \\
\cline { 2 - 9 } & tp & tn & $\mathbf{f p}$ & $\mathbf{f n}$ & Sens & Spec & Acc & MCC \\
\hline PSLNuc & 2,317 & 2,030 & 576 & 525 & $\mathbf{0 . 8 1 5}$ & $\mathbf{0 . 7 7 9}$ & $\mathbf{0 . 7 9 7}$ & $\mathbf{0 . 5 9 5}$ \\
NUCLEO & 2,157 & 1,924 & 682 & 685 & 0.759 & 0.760 & 0.749 & 0.497 \\
\hline \multicolumn{7}{c}{ Independent Test Data Set } \\
PSLNuc & 452 & 258 & 140 & 112 & $\mathbf{0 . 8 0 5}$ & $\mathbf{0 . 6 4 6}$ & $\mathbf{0 . 7 3 9}$ & $\mathbf{0 . 4 5 7}$ \\
NUCLEO & 430 & 246 & 152 & 134 & 0.760 & 0.620 & 0.700 & 0.380 \\
PredictNLS & 153 & 369 & 29 & 411 & 0.270 & 0.930 & 0.540 & 0.250 \\
NucPred & 376 & 233 & 165 & 188 & 0.670 & 0.590 & 0.630 & 0.250 \\
\hline Pern & tp & fp & fn & Sens & Spec & Acc & MCC \\
\hline
\end{tabular}

Performance comparison of different nuclear localization predictors. 
proteins are listed in Table 3 and Table 4, respectively. We compare the generated gapped-dipeptide signatures with known experimentally determined NLSs and NESs in the biological literature and databases. The signatures that have been reported as motifs for NLSs and NESs are shown in bold face in Table 3 and Table 4, respectively. It is observed that many gapped-dipeptide signatures are motifs critical for predicting nuclear localization, especially for NLSs. Our experiment results show that the proposed method can capture biological features of nuclear and non-nuclear proteins.

Figure 1(A) and 1(B) illustrate further analyses of amino acid composition and grouped amino acid composition of selected gapped-dipeptide signatures, respectively. When analyzing physicochemical properties in grouped amino acid composition, each amino acid is grouped into one of the four categories: aromatic (FYW), charged (DEHKR), nonpolar (AIGLMV), and polar (CNPQST). From Figure 1(A), it is observed that nuclear signatures show great preferences for Arginine $(\mathrm{R})$, Histidine $(\mathrm{H})$, and Lysine $(\mathrm{K})$, which can be discovered in most NLSs; while non-nuclear signatures exhibit high compositions in Leucine (L), Isoleucine (I), Phenylalanine (F), Valine (V), Methionine (M), and Proline (P), which occur frequently in NESs. In addition, as shown in Figure 1(B), nuclear signatures prefer basic (HKR) or polar (CGNQSTY) amino acids, and nonnuclear ones favor hydrophobic (AFILMPVW) amino acids. Experiment results demonstrate that the amino

Table 3 Gapped-dipeptide signatures for nuclear proteins

\begin{tabular}{cccccccccc}
\hline \multicolumn{6}{c}{ Gapped-dipeptide signatures for nuclear proteins } \\
\hline G2P & G11P & G8P & P11P & P5P & P3Q & P5Q & K1K & K6K & K10K \\
K2K & K5K & K0K & K3K & K4K & E4E & E1E & E8E & E0E & P1T \\
P0T & P6T & P10T & G2G & G1G & G5G & P1S & S6P & P3G & G4P \\
P0G & Q11Q & Q10Q & Q5Q & R13R & R11R & R10R & R9R & R12R & R7R \\
R3R & R4R & C8S & S2C & S6C & N6P & P12N & S9D & E12D & D12D \\
D4D & D13D & S12T & S8T & S10T & K0R & K2R & R2R & R0R & R0K \\
R2K & K1R & R1K & R3K & K4R & M3P & P12M & R1H & Q8R & Q2H \\
H0R & S7Q & Q12S & S13H & S10H & S2H & H6S & S12H & P7Y & P4Y \\
Y5P & N3N & N6N & N0N & K1H & H0K & H6K & K10H & D2S & D5S \\
K8S & D0S & N8N & H8N & H5N & S6L & T4E & E13S & T6E & S13S \\
S10S & H0H & H4H & H1H & H13H & N0E & N0K & N4K & K1N & D2T \\
G6L & G8L & E8C & K1C & K5C & K7C & C2C & C0C & K3C & C4K \\
H8C & H12C & H13C & H10C & D6I & D10I & D121 & A13Q & A6Q & Q9A \\
Q6A & S11S & M3S & Q13T & K9T & Q1T & Q9T & N1S & S7N & N8S \\
H1I & IOH & E12I & S2E & E11I & D6A & S3D & D8A & M13Q & M3Q \\
L13S & L12S & V6S & S9N & S13G & E9S & S3S & N0S & S3N & H1Q \\
H10Q & H3Q & H5Q & T7E & T8E & T12E & T9E & W13K & W11K & N11I \\
I12N & M13D & M2D & F13D & L1H & H1L & A3H & L5H & R0G & A9H \\
H10A & P3R & A10H & & & & & & & \\
\hline Our & & & & & & & \\
\hline
\end{tabular}

Our method propose 183 gapped-dipeptide signatures for nuclear proteins. Signatures that have been reported as motifs for nuclear localization signals (NLSs) are shown in bold face.
Table 4 Gapped-dipeptide signatures for non-nuclear proteins

\begin{tabular}{|c|c|c|c|c|c|c|c|c|c|}
\hline \multicolumn{10}{|c|}{ Gapped-dipeptide signatures for non-nuclear proteins } \\
\hline L2L & L2F & F5L & F2L & MOV & M1V & VoM & V1M & V3V & V2V \\
\hline $\mathrm{A} 3 \mathrm{~V}$ & A8V & A121 & A6l & $\mathrm{A} 4 \mathrm{I}$ & $12 \mathrm{~A}$ & E11L & L11Q & Q11L & L11E \\
\hline Q5W & Q13W & S7W & Q12W & $|13|$ & 191 & 1121 & $|10|$ & Y6Y & YOY \\
\hline Y5Y & $\mathrm{Y} 12 \mathrm{Y}$ & VON & L2N & $12 \mathrm{Q}$ & $12 \mathrm{~S}$ & $\mathrm{R} 11 \mathrm{~A}$ & R8A & R1A & $\mathrm{K} 1 \mathrm{~L}$ \\
\hline L1K & LOK & F10A & F11A & F11S & F10S & L8A & L4A & L5A & $\mathrm{A} 6 \mathrm{~L}$ \\
\hline F3F & F4F & F10F & F13F & L8L & L3L & I3L & L12L & V11D & V13D \\
\hline V7D & V4D & I5E & $14 \mathrm{D}$ & I12E & E5I & W8P & W9P & W10P & W3P \\
\hline V5V & V13V & G2V & G11V & $\mathrm{S} 2 \mathrm{~S}$ & S4S & A5S & S9S & $\mathrm{W} 1 \mathrm{H}$ & $\mathrm{H} 10 \mathrm{~W}$ \\
\hline W8H & Y10W & M3M & $\mathrm{M} 2 \mathrm{M}$ & M13M & MOM & TOT & $\mathrm{T} 13 \mathrm{~T}$ & T7T & $\mathrm{T} 1 \mathrm{~T}$ \\
\hline $\mathrm{H} 1 \mathrm{Y}$ & $\mathrm{H} 3 \mathrm{Y}$ & $\mathrm{H} 13 \mathrm{Y}$ & $\mathrm{H} 7 \mathrm{Y}$ & $\mathrm{F} 12 \mathrm{C}$ & F9C & FOC & F10C & A7K & A3K \\
\hline $\mathrm{A} 10 \mathrm{~A}$ & $\mathrm{~A} 4 \mathrm{~A}$ & A5A & A9A & Y2G & YOG & GOY & GOF & F2K & KOF \\
\hline K3F & Q13L & L13Q & L1T & L10Q & $\mathrm{C} 121$ & $112 \mathrm{C}$ & $\mathrm{C} 10 \mathrm{l}$ & C3I & W4E \\
\hline WOL & W5L & W1L & Y7T & Y7V & Y6V & V5Y & E1M & L9K & $\mathrm{R} 11 \mathrm{M}$ \\
\hline $110 \mathrm{~K}$ & $113 \mathrm{~K}$ & F13K & T1K & K5I & $\mathrm{K} 2 \mathrm{~V}$ & $\mathrm{H} 10 \mathrm{~L}$ & $\mathrm{~L} 12 \mathrm{H}$ & $\mathrm{H} 8 \mathrm{~L}$ & E13L \\
\hline E13I & E10V & N13L & $\mathrm{K} 13 \mathrm{~N}$ & $\mathrm{~K} 12 \mathrm{~N}$ & I9N & V10N & $\mathrm{V} 12 \mathrm{~N}$ & $\mathrm{~V} 1 \mathrm{~N}$ & R10Q \\
\hline D3R & R2D & V7G & $\mathrm{M} 11 \mathrm{G}$ & V13G & L3G & $16 \mathrm{~N}$ & $15 \mathrm{~N}$ & V9l & $111 \mathrm{~N}$ \\
\hline K7L & $\mathrm{V} 4 \mathrm{H}$ & $\mathrm{H} 12 \mathrm{~V}$ & $\mathrm{~V} 8 \mathrm{H}$ & $\mathrm{H} 4 \mathrm{~V}$ & F9E & D13L & D12L & L3D & D9L \\
\hline $\mathrm{H} 11 \mathrm{M}$ & L10M & M7H & $\mathrm{H} 12 \mathrm{I}$ & E9L & E11A & E12A & A5E & E10A & $14 \mathrm{~N}$ \\
\hline $\mathrm{NOI}$ & N13I & N9l & & & & & & & \\
\hline
\end{tabular}

Our method propose 183 gapped-dipeptide signatures for non-nuclear proteins. Signatures that have been reported as motifs for nuclear export signals (NESs) are shown in bold face.

acid preferences of selected nuclear and non-nuclear signatures correspond well with biological knowledge [15].

Gapped-dipeptide signatures better discriminate nuclear and non-nuclear proteins

To demonstrate that our method can capture biological properties of putative NLSs and NESs, we further examine the predictive performance using only the proposed gapped-dipeptide signatures. In PSLNTS, we incorporate 366 selected gapped-dipeptide signatures in an SVM classifier to see whether gapped-dipeptide signatures can better discriminate nuclear and nonnuclear proteins. Table 5 compares the performance of PSLNTS, PSLNuc, and NUCLEO. Using only the 366 gapped-dipeptide signatures, PSLNTS performs slightly better than PSLNuc by nearly 0.010 (1\%) and 0.023 for overall accuracy and $M C C$, respectively. Experiment results demonstrate that the selected signatures can capture biological properties of NLSs and NESs, and thus, can resolve the ambiguity to discriminate nuclear and non-nuclear proteins.

\section{Conclusions}

In this study, we first incorporate gapped-dipeptides weighted by a smoothPSSM encoding and reduced by PLSI to predict nuclear localization in PSLNuc. Our results show that PSLNuc significantly improves the 
(A)

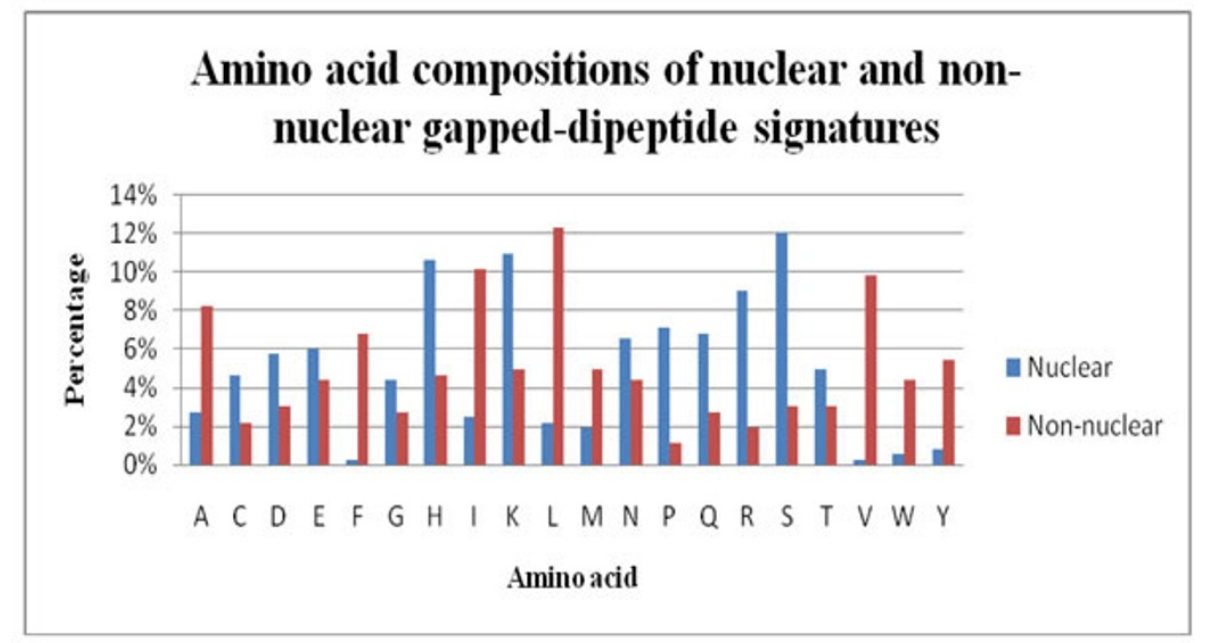

(B)

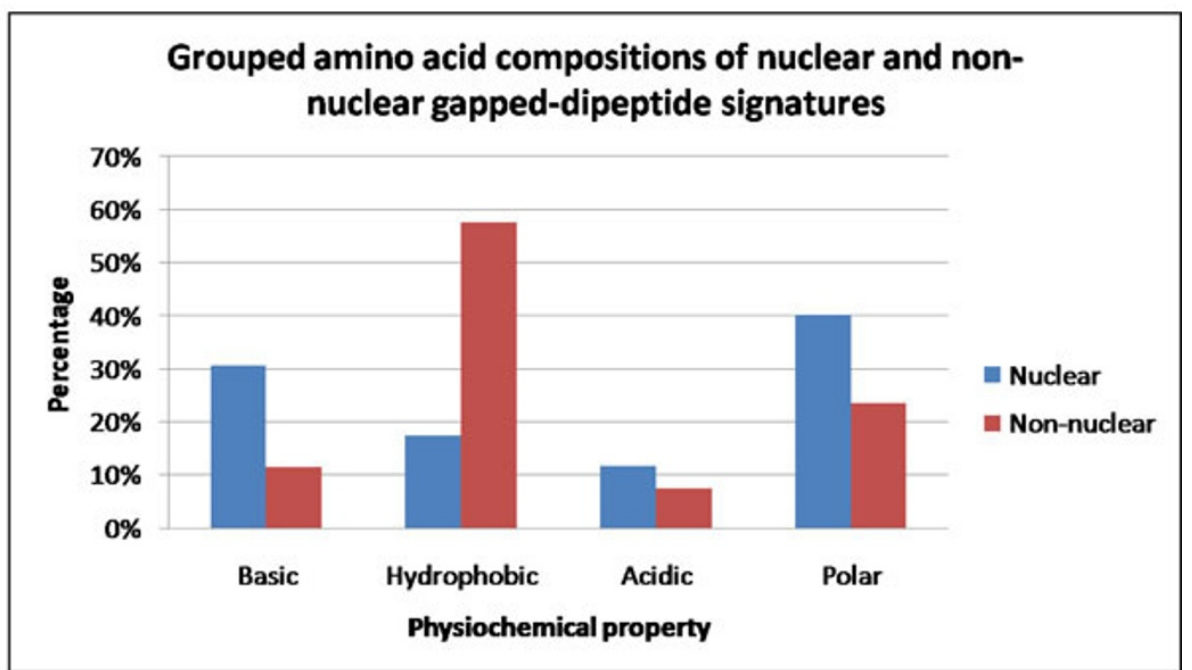

Figure 1 Physicochemical analyses of gapped-dipeptide signatures. (A) Amino acid compositions and (B) grouped amino acid compositions of gapped-dipeptide signatures for nuclear and non-nuclear proteins.

predictive performance compared to the state-of-the-art system. Experiment results also suggest that, in addition

Table 5 Performance comparison using gapped-dipeptide signatures

\begin{tabular}{lcccccccc}
\hline & \multicolumn{8}{c}{ Training Set (by five-fold cross-validation) } \\
\cline { 2 - 9 } & tp & tn & fp & fn & Sens & Spec & Acc & MCC \\
\hline PSLNTS & 2,429 & 1,978 & 628 & 413 & $\mathbf{0 . 8 5 5}$ & $\mathbf{0 . 7 5 9}$ & $\mathbf{0 . 8 0 9}$ & $\mathbf{0 . 6 1 8}$ \\
PSLNUC & 2,413 & 1,964 & 642 & 429 & 0.815 & 0.779 & 0.797 & 0.595 \\
NUCLEO & 2,157 & 1,924 & 682 & 685 & 0.759 & 0.760 & 0.749 & 0.497 \\
\hline
\end{tabular}

Performance comparison of PSLNUC, PSLNTS, and NUCLEO. to NLSs, which have been shown important for nuclear proteins, NESs can also be an effective indicator to detect non-nuclear proteins. Secondly, we apply only a few proposed gapped-dipeptide signatures in PSLNTS and further enhance the accuracy and MCC to 0.809 and 0.618 , respectively. This demonstrates that gappeddipeptide signatures can better discriminate nuclear and non-nuclear localization. Most notably, the proposed gapped-dipeptide signatures could be biologically interpreted and used in further experiment studies of nuclear translocation sequences, including NLSs and NESs. 


\section{Methods}

In this study, we extend our previously proposed method for general localization site prediction [16] and formulate the nuclear protein prediction as a document classification problem. In our previous work, we incorporated gapped-dipeptides as feature representation and PLSI as feature reduction for predicting protein localization sites. The method was inspired by word representation and word vector reduction in the research of document classification, where a document is assigned to one or several categories according to its content. Similarly, prediction of nuclear localization can be formulated as a document classification problem, in which a protein sequence is regarded as a document's content and its subcellular localization classes can be treated as categories of the document. Classification of documents is often solved in steps described as follows. First, for feature representation, each document is represented by a feature vector, where each word denotes a feature and its feature value represents the weight of a word in the document. Second, due to a high-dimensional feature space of words in a document, features are further reduced to enhance prediction accuracy and prevent overestimation [17]. Finally, reduced features are incorporated as input vectors in machine learning approaches for predicting document categories. To calculate the weights of each word, a standard PSSM encoding was incorporated to predict general protein localization in our previous study [16]. However, it has been shown that a smoothPSSM encoding scheme is more effective to predict protein structure and function [18]. In this study, we incorporate a new smoothPSSM encoding scheme and extend our approach to predict nuclear localization for bioinformatics analysis. The task is a large-scale analysis of nuclear proteins, including prediction of nuclear localization and analyses of nuclear translocation signals. To solve these problems, we propose a prediction method in which proteins are represented by gapped-dipeptides from smoothPSSM and PLSI is incorporated for feature reduction. Next, the feature representation, feature weighting, feature reduction, system architecture, and evaluation measures are described in the following sections.

\section{Feature representation - gapped-dipeptides as words of proteins}

When proteins are considered as documents, several types of word representation have been used, such as amino acid compositions [19] and $k$-peptide compositions [20]. Specifically, a dipeptide $(k=2)$ composition can be considered as a bi-gram word representation. However, peptides with gaps cannot be represented by a $k$-peptide composition. In addition, feature vectors with high dimensions could be generated if $k$-peptide compositions are used to represent remote sequence information. For instance, the dimension of a feature vector reaches up to 8,000 if a tri-peptide composition is considered. To distinguish nuclear and non-nuclear proteins, we incorporate gappeddipeptide representation used in our previous work [16] to represent sequence features in proteins. A gapped-dipeptide $A d B$ represents the sequence patterns that two amino acids $A$ and $B$ are divided by $d$ residues. When we consider gapped-dipeptides up to $u$ gapped distance, the feature dimension of gapped-dipeptides in a protein is the total number of probable combinations, that is $20 \times 20 \times$ $(u+1)$. For instance, when $u=16$, a feature vector of 6,400 $(=20 \times 20 \times 16)$ dimension is used to represent a protein sequence. Due to computational and time complexity, tripeptides or other $k$-peptides are not considered in this study.

\section{Feature weighting - homology information from position-specific scoring matrix Standard position-specific scoring matrix}

As shown in Figure 2(A), a standard PSSM in a protein $P$ of $n$ amino acids is denoted as an $n \times 20$ matrix, where $n$ denotes the residues in protein $P$ and 20 represents the twenty amino acids. The elements in the standard PSSM denote the amino acid substitution log-likelihood of different residues in the protein $P$ [21]. We incorporate PSIBLAST to generate homology information from PSSM. The parameters in PSI-BLAST are set to $j=3$ (three iterations), $e=10^{-3}$, and the searched database is NCBI nr.

\section{Smoothed position-specific scoring matrix}

In this study, we incorporate a smoothPSSM encoding scheme, which has been shown effective for proteinRNA binding site prediction by considering the dependency or correlation among neighboring residues [18]. In a standard PSSM profile, the log-likelihood at each position is calculated based on an assumption that each position is independent from the others. Inspired by the consideration of adjacent pixels used in the spatial domain method from the research field of image processing, a new encoding scheme is proposed to consider the dependency among surrounding neighbors. We use a sliding window of size $w$ to incorporate the homology information from upstream and downstream residues. In the construction of a smoothPSSM, each row vector of a residue $\alpha_{i}$ is represented and smoothed by the summation of $w$ surrounding row vectors $\left(V_{\text {smoothed } i}=\right.$ $\left.V_{i-(w-1) / 2}+\ldots+V_{i}+\ldots+V_{i+(w-1) / 2}\right)$. For the $\mathrm{N}$-terminal and C-terminal of a protein, $(w-1) / 2$ zero vectors are appended to the hand or tail of a smoothPSSM profile. Using the smoothPSSM encoding scheme, the feature vector of a residue $\alpha_{i}$ is represented by ( $V_{\text {smoothed_i-(w-1) }}$ $\left.{ }_{2}, \ldots, V_{\text {smoothed_i } i}, \ldots, V_{\text {smoothed_i+(w-1)/2 }}\right)$. Here, we adopt gapped-distance as 13 and smoothing window sizes as 7 according to our previous studies $[16,18]$. Figure 2(B) 


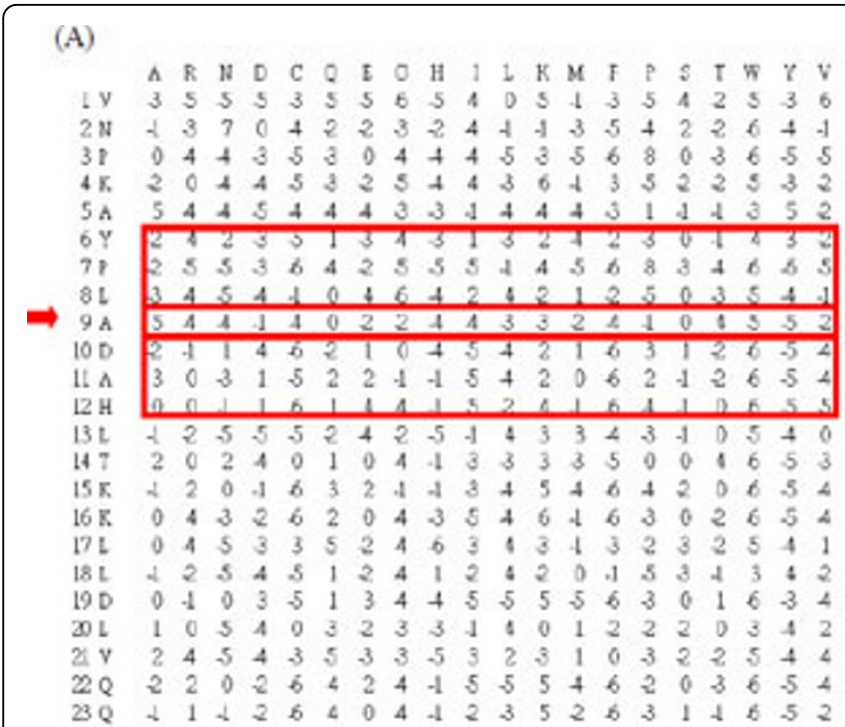

(B)

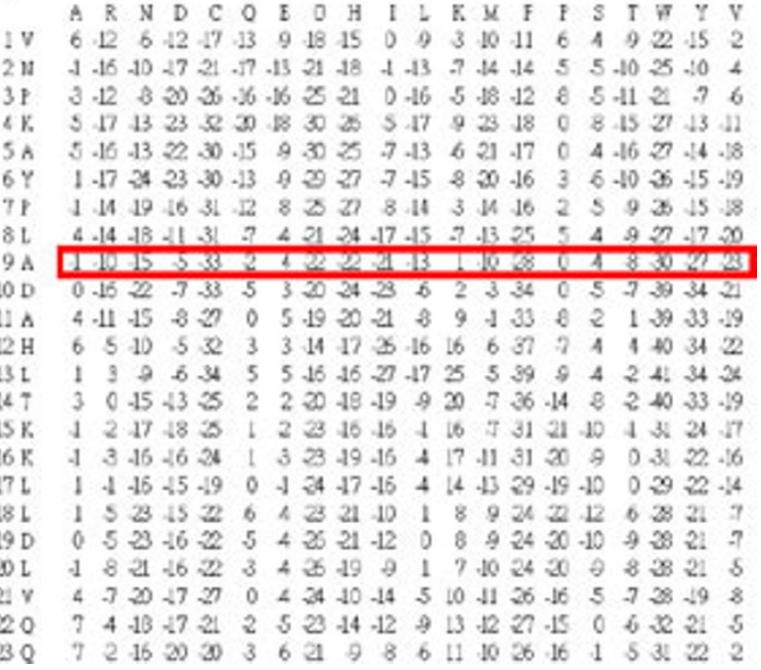

Figure 2 Construction of a smoothed PSSM profile. Transformation of (A) a standard PSSM profile into (B) a smoothed PSSM profile using $W$ as 7 .

illustrates an example of a smoothPSSM profile. At position 9, the corresponding value of amino acid 'A' represented by a smoothPSSM encoding is the sum of $[(-2)+$ $(-2)+(-3)+5+(-2)+3+0]$. The log-likelihoods in a smoothPSSM are normalized to $0 \sim 1$ based on a logistic function [22]:

$$
f(x)=\frac{1}{1+e^{-x}}
$$

where $x$ is a log-likelihood in a smoothPSSM.

\section{TFsmoothPSSM weighting scheme}

Using smoothPSSM, a TFsmoothPSSM encoding is calculated in the following steps. For a protein sequence $P$ of length $n$, a gapped-dipeptide $\mathrm{A} d \mathrm{~B}$ of $P$ contains smoothPSSM elements that corresponds to sequence pattern $P_{i} d P_{i+d+1}$ for $1 \leqq i \leqq n-(d+1)$, in which $P_{i}$ represents the $i$ th amino acid of $P$. Take a protein sequence MKGIKSKMLS as an example. The gapped-dipeptide M2I only shows one occurrence in the protein. However, the weighting of M2I can be contributed from different gapped-dipeptides of the protein in a smoothPSSM (i.e., M2I, K2K, G2S, I2K, K2M, S2L, and K2S). The word weighting of a gapped-dipeptide $\mathrm{A} d \mathrm{~B}$ in $P$ is calculated by

$$
W(A d B, P)=\sum_{1 \leq i \leq n-(d+1)} s f(i, A) \times s f(i+d+1, B)
$$

in which $s f(i, A)$ represents a normalized log-likelihood in the smoothPSSM element of the $i$ th row and the $A$ th column. From the sample sequence, the weighting of M2I using a smoothPSSM profile is calculated as $s f(1, \mathrm{M}) \times s f$
$(4, \mathrm{I})+s f(2, \mathrm{M}) \times s f(5, \mathrm{I})+\ldots+s f(7, \mathrm{M}) \times s f(10, \mathrm{I})$. A protein is denoted as a feature vector consisting of gappeddipeptides, where each gapped-dipeptide is weighted by TFsmoothPSSM encoding scheme. Finally, the feature vector is normalized to a range of 0 to 1 .

Feature reduction - probabilistic latent semantic indexing Probabilistic latent semantic indexing

Hofmann proposed PLSI based on an aspect model for feature reduction [17]. PLSI aims at identifying and discriminating between different contexts of word usage without the help from a thesaurus or dictionary. It can identify semantic similarities by classifying together words with identical or similar meanings. Latent topic variables $\mathrm{t} \in T=\left\{t_{1}, \ldots, t_{K}\right\}$ for co-occurrence data are incorporated to associate each observation in the aspect model. We use a latent topic variable $t$ to model the weight of a word $w$ in a document $d$, which is regarded as a joint probability $P(w, d)$ between $w$ and $d$. Therefore, $P(w, d)$ is calculated by

$$
P(w, d)=P(d) P(w \mid d), P(w \mid d)=\sum_{t \in T} P(w \mid t) P(t \mid d)
$$

in which $P(w \mid t)$ represents the conditional probability of a word $w$ conditioned on a topic $t$, and $P(t \mid d)$ represents the weighting of a topic variable $t$ in a document $d$. It is assumed that the word distribution given a topic class is conditionally independent of the document $d$, i. e., $P(w \mid t, d)=P(w \mid t)$. Therefore, the original feature dimension $|W|$ of the word vector is greatly reduced to the number of latent topic variables $|T|$. 


\section{Probabilistic latent semantic indexing training and testing}

We incorporate the same procedure as described in our previous work [16] to train and test PLSI model. First, for PLSI training, the parameters $P(w \mid t)$ and $P(t \mid d)$ are fitted by an iterative expectation-maximization algorithm, in which $P(t \mid d)$ is estimated in the expectation (E) step and $P(w \mid t)$ is recalculated in the maximization (M) step. Then, after training, the calculated probability of a word conditioned on a topic $P(w \mid t)$ is used to estimate the $P\left(t \mid d^{\prime}\right)$ for new documents $d^{\prime}$ through a folding-in process [17] in PLSI testing.

\section{Feature reduction by probabilistic latent semantic indexing}

The application of PLSI can not only reduce feature dimension, but also extract semantic relationships of gapped-dipeptides. During PLSI feature reduction process, gapped-dipeptides with similar meanings or preferences are grouped together in a semantic topic, and then the topic preferences to nuclear or non-nuclear localization can be identified. If we can select an appropriate topic size in feature reduction, the mappings of feature vectors from the gapped-dipeptide space to latent semantic topic space can greatly increase the learning efficiency and performance. One way to approximate the reduced feature size is based on latent semantic indexing (LSI). First, singular values of LSI are calculated and sorted in a decreasing order. After that, we select $t$ as the reduced feature size of LSI if the $t$-th largest singular value is close to zero. Although PLSI is not identical to LSI, the number of singular values larger than zero is reasonably estimated by the number of the PLSI reduced dimensions. We take the reduced number of topics as 80 according to our previous study [16].

\section{System architecture}

Prediction of protein nuclear localization can be regarded as a two-class classification problem. For a two-class classification problem, the SVM classifier has been demonstrated effective in classification [23]. Our prediction method consists of a one-versus-one SVM classifier corresponding to nuclear and non-nuclear proteins. For the SVM classifier in our system, we incorporate libsvm [23], where probability estimation values are shown to determine the classification confidences [24]. In libsvm, we use the Radial Basis Function (RBF) kernel and tune cost $(c)$ and gamma $(\gamma)$ parameters based on five-fold cross-validation. Here we propose PSLNuc for predicting nuclear proteins using smoothPSSM and PLSI. Our method applies gapped-dipeptides weighted by TFsmoothPSSM scheme to represent features of a protein. After that, the feature vectors are reduced by PLSI and incorporated as input vectors for an SVM classifier. The system architecture of PSLNuc is illustrated in Figure 3. For each protein, PSLNuc predicts its localization according to these steps:
1. Perform PSI-BLAST to calculate a standard PSSM for the protein.

2. Construct a smoothPSSM profile based on the standard PSSM.

3. Use gapped-dipeptides to represent the protein and incorporate TFsmoothPSSM encoding scheme to determine the weights in a feature vector.

4. Apply PLSI to reduce the feature vector.

5. Use the reduced feature vector as input and run the one-versus-one (nuclear and non-nuclear) SVM classifier.

\section{Nuclear protein prediction based on proposed translocation signals}

For localization-topic preference identification, we divide the training data sets into nuclear and non-nuclear proteins to examine preferred topics. The localization-topic preference of a topic is computed as the average of topic weights from the proteins in a localization site. A topic is identified as showing preference to a localization site if its localization-topic preference is larger than the other site. For nuclear localization prediction, we divide the proteins into two categories, and select 10 top preferred topics for nuclear and non-nuclear proteins according to localization-topic preference, respectively. To list gapped-dipeptides of interest, for each topic, up to 20 (depending on the number gapped-dipeptides in the topic) most frequent gapped-dipeptides are selected. After that, we incorporate only the proposed gappeddipeptide signatures for nuclear and non-nuclear proteins for predicting nuclear localization. We apply the proposed gapped-dipeptide signatures to capture biological properties and mimic translocation mechanisms of nuclear translocation.

\section{Evaluation measures}

For predictive performance comparison, the same evaluation measures applied in other approaches [2-5] are incorporated. Evaluation measures include sensitivity $($ Sens), specificity (Spec), accuracy (Acc; also known as success rate), and MCC defined in Equation (4), (5), (6), and (7) below:

$$
\begin{aligned}
& \text { Sensitivity }=\frac{T P}{T P+F N} \\
& \text { Specificity }=\frac{T N}{T N+F P} \\
& \text { Accuracy }=(T P+T N) / N \\
& M C C=\frac{T P \times T N-F P \times F N}{\sqrt{(T P+F N)(T P+F P)(T N+F P)(T N+F N)}}
\end{aligned}
$$




\section{MPLDLYNTLT...}

\section{Smoothed PSSM encoding based on PSSM generated from PSI-BLAST}
A R N D C Q E G H I L K M F P T W Y V
$1 \mathrm{M}-3-3-4-5-3-3-4-5-4 \quad 0 \quad 1-3 \quad 10-2-5-4-3-4-3-1$

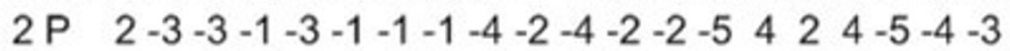

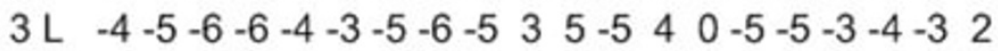

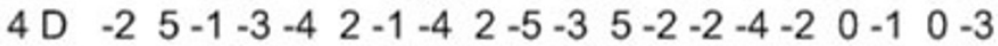

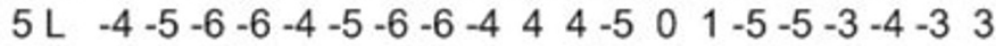

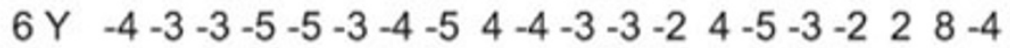
$7 \mathrm{~N}-4-3 \quad 8 \quad 4-6-3-2-3-2-6-6-3-5-6-4-1-3-7-5-6$
$8 \mathrm{~T}-2-3-1-3-1-3-3-4-3-4-4-1-4-4-4-4 \quad 4 \quad 6-5-4-2$

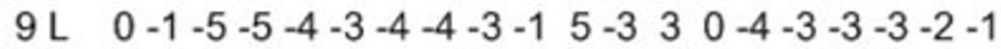
10 T $-1-3-1-1-4-2-3-2-1-4-3-1-3-4-4 \quad 3 \quad 6-5-4-3$

\section{Gapped-Dipeptides Representation}

A0A, A1A, A2A, A3A, A4A, A5A, $\cdots, \quad$ Y5Y

$\{0.81396,0.78755,0.788206,0.799535,0.784058,0.742093, \cdots, 0.437457\}$

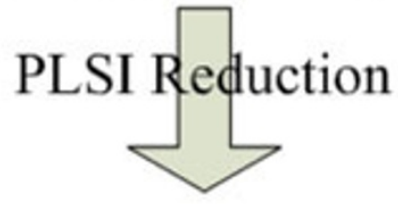

$\{0.012103,0.014095,0.015480,0.018894, \cdots, 0.003121\}$

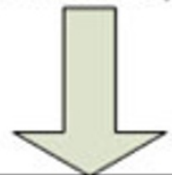

\section{Support Vector Machines (SVM)}

where $T P, T N, F P, F N$, and $N$ represent the number of true positives, true negatives, false positives, false negatives, and total number of protein sequences, respectively.
For an objective comparison with other approaches that use five-fold cross-validation, we also apply five-fold cross-validation to evaluate our predictive performance. 


\section{Additional material}

Additional file 1: Data sets. The protein sequences in the benchmark data sets used for training and testing are listed as supplementary data.

\section{Acknowledgements}

We thank Hua-Sheng Chiu and Allan Lo for helpful suggestions and computational assistance. The research was supported in part by Taipei Medical University under grant TMU98-AE1-B05 and National Science Council under grant NSC99-2218-E-038-002 and NSC100-2221-E-038-012 to Emily Chia-Yu Su. JMC is funded by "la Caixa" pre-dotocorl fellowship and the Centre de Regulacio Genomica (CRG), the Plan Nacional (BFU200800419) from the Spanish Ministry of Science.

This article has been published as part of BMC Bioinformatics Volume 13 Supplement 17, 2012: Eleventh International Conference on Bioinformatics (InCoB2012): Bioinformatics. The full contents of the supplement are available online at http://www.biomedcentral.com/bmcbioinformatics/ supplements/13/S17.

\section{Author details}

${ }^{1}$ Graduate Institute of Biomedical Informatics, Taipei Medical University, Taipei, Taiwan. ${ }^{2}$ Comparative Bioinformatics, Bioinformatics and Genomics, Centre for Genomic Regulation (CRG), Barcelona, 08003, Spain. ${ }^{3}$ Universitat Pompeu Fabra (UPF), Barcelona, 08003, Spain. ${ }^{4}$ Bioinformatics Lab., Institute of Information Science, Academia Sinica, Taipei, Taiwan.

\section{Authors' contributions}

ECYS developed the method, carried out the computational predictions, and drafted the manuscript. JMC and CWC participated in the experimental design and supplied additional insights regarding the analyses. TYS and WLH refined the manuscript. All authors read and approved the final manuscript.

\section{Competing interests}

The authors declare that they have no competing interests.

Published: 13 December 2012

\section{References}

1. Macara IG: Transport into and out of the nucleus. Microbiol Mol Biol Rev 2001, 65(4):570-594, table of contents.

2. Cokol M, Nair R, Rost B: Finding nuclear localization signals. EMBO Rep 2000, 1(5):411-415.

3. Brameier $M$, Krings $A$, MacCallum RM: NucPred-predicting nuclear localization of proteins. Bioinformatics 2007, 23(9):1159-1160.

4. Hawkins J, Davis L, Boden M: Predicting nuclear localization. J Proteome Res 2007, 6(4):1402-1409.

5. Kumar M, Raghava GPS: Prediction of nuclear proteins using SVM and HMM models. BMC Bioinformatics 2009, 10:22.

6. Guda C, Fahy E, Subramaniam S: MITOPRED: a genome-scale method for prediction of nucleus-encoded mitochondrial proteins. Bioinformatics 2004, 20(11):1785-1794.

7. Nguyen Ba AN, Pogoutse A, Provart N, Moses AM: NLStradamus: a simple Hidden Markov Model for nuclear localization signal prediction. BMC Bioinformatics 2009, 10:202.

8. la Cour T, Kiemer L, Molgaard A, Gupta R, Skriver K, Brunak S: Analysis and prediction of leucine-rich nuclear export signals. Protein Eng Des Sel 2004, 17(6):527-536.

9. Scott MS, Troshin PV, Barton GJ: NoD: a nucleolar localization sequence detector for eukaryotic and viral proteins. BMC Bioinformatics 2011, 12:317.

10. Huang WL, Tung CW, Huang HL, Hwang SF, Ho SY: ProLoc: prediction of protein subnuclear localization using SVM with automatic selection from physicochemical composition features. Biosystems 2007, 90(2):573-581.

11. Lei Z, Dai Y: An SVM-based system for predicting protein subnuclear localizations. BMC Bioinformatics 2005, 6:291.

12. Lei $Z$, Dai $Y$ : Assessing protein similarity with Gene Ontology and its use in subnuclear localization prediction. BMC Bioinformatics 2006, 7:491.
13. Shen HB, Chou KC: Nuc-PLoc: a new web-server for predicting protein subnuclear localization by fusing PseAA composition and PsePSSM. Protein Eng Des Sel 2007, 20(11):561-567.

14. Su EC, Chiu HS, Lo A, Hwang JK, Sung TY, Hsu WL: Protein subcellular localization prediction based on compartment-specific features and structure conservation. BMC Bioinformatics 2007, 8:330

15. Christophe D, Christophe-Hobertus C, Pichon B: Nuclear targeting of proteins: how many different signals? Cell Signal 2000, 12(5):337-341.

16. Chang JM, Su EC, Lo A, Chiu HS, Sung TY, Hsu WL: PSLDoc: Protein subcellular localization prediction based on gapped-dipeptides and probabilistic latent semantic analysis. Proteins 2008, 72(2):693-710.

17. Hofmann T: Unsupervised learning by probabilistic latent semantic analysis. Mach Learn 2001, 42(1-2):177-196.

18. Cheng CW, Su EC, Hwang JK, Sung TY, Hsu WL: Predicting RNA-binding sites of proteins using support vector machines and evolutionary information. BMC Bioinformatics 2008, 9(Suppl 12):S6.

19. Hua S, Sun Z: Support vector machine approach for protein subcellular localization prediction. Bioinformatics 2001, 17(8):721-728.

20. Yu CS, Lin CJ, Hwang JK: Predicting subcellular localization of proteins for Gram-negative bacteria by support vector machines based on n-peptide compositions. Protein Sci 2004, 13(5):1402-1406.

21. Altschul SF, Madden TL, Schaffer AA, Zhang JH, Zhang Z, Miller W, Lipman DJ: Gapped BLAST and PSI-BLAST: a new generation of protein database search programs. Nucleic Acids Research 1997, 25(17):3389-3402.

22. Jones DT: Protein secondary structure prediction based on positionspecific scoring matrices. J Mol Biol 1999, 292(2):195-202.

23. Chang CC, Lin CJ: LIBSVM: a library for support vector machines. $A C M$ Transactions on Intelligent Systems and Technology 2011, 2(27).

24. Wu TF, Lin CJ, Weng RC: Probability estimates for multi-class classification by pairwise coupling. J Mach Learn Res 2004, 5:975-1005.

doi:10.1186/1471-2105-13-S17-S13

Cite this article as: Su et al.: Prediction of nuclear proteins using nuclear translocation signals proposed by probabilistic latent semantic indexing. BMC Bioinformatics 2012 13(Suppl 17):S13.

\section{Submit your next manuscript to BioMed Central and take full advantage of:}

- Convenient online submission

- Thorough peer review

- No space constraints or color figure charges

- Immediate publication on acceptance

- Inclusion in PubMed, CAS, Scopus and Google Scholar

- Research which is freely available for redistribution

Submit your manuscript at www.biomedcentral.com/submit
C Biomed Central 